\title{
Antarctic site testing: First daytime seeing monitoring at Dome C
}

\author{
E. Aristidi, A. Agabi, J. Vernin, M. Azouit, F. Martin, A. Ziad, and E. Fossat \\ Laboratoire Universitaire d'Astrophysique de Nice, Université de Nice Sophia Antipolis, Parc Valrose, 06108 Nice Cedex 2 , \\ France
}

Received 22 April 2003 / Accepted 4 June 2003

\begin{abstract}
The first astronomical seeing monitoring has been made with a DIMM instrument at the Antarctic plateau site of Dome $\mathrm{C}$ in December, 2002 on the bright star Canopus ( $\alpha$ Eri) during the daytime. In these far from optimal conditions, a median seeing value of 1.20 arcsec as been obtained, with extended periods better than 1 arcsec and 12 percent of the time better than 0.75 arcsec.
\end{abstract}

Key words. site testing

\section{Introduction}

The French (IPEV) and Italian (ENEA) polar Institutes are constructing the Concordia base on the Dome $\mathrm{C}$ site of the polar plateau $\left(75^{\circ} \mathrm{S}, 123^{\circ} \mathrm{E}\right)$, at an elevation of $3250 \mathrm{~m}$, that corresponds, given the cold air temperatures, to an air pressure encountered around $3800 \mathrm{~m}$ at more standard latitudes. The Concordia construction is now near completion, the first winterover being scheduled for 2004. Astronomy is obviously near the top of the list of the scientific programmes that will benefit of this unique site: the extremely cold and dry air is probably complemented by very low winds, both at ground level and at higher altitude, so that an exceptionally good seeing is expected. That remains to be demonstrated, and the first DIMM instrument (Sarazin \& Roddier 1990) as been tested for this purpose during the summer season 2002/2003. The exceptional transparency of the Antarctic sky made possible to measure the seeing on a bright star at any time of the day, even with the Sun being at an elevation angle of $38^{\circ}$.

\section{Instrumentation}

The differential image motion monitor (DIMM) measures the differential motion of two stellar images at the focus of a small telescope. A mask with two sub-apertures is located at the entrance pupil. A prism is added on one of the sub-apertures, so that two images of the same star are observed at the focus. The differential motion of these two images is related to the Fried parameter. Our DIMM is based upon the instrumental concept described by Vernin \& Munoz (1995). The instrumental set up was as follows:

- Celestron 11 telescope ( $28 \mathrm{~cm}$ aperture, $2.8 \mathrm{~m}$ focus) on an Astrophysics 1200 equatorial mount

Send offprint requests to: E. Aristidi, e-mail: aristidi@unice.fr
- $6 \mathrm{~cm}$ diameter sub-apertures separated by $20 \mathrm{~cm}$. The mask was placed at the top of the telescope, $5 \mathrm{~cm}$ above the Schmidt entrance corrective window

- A glass prism of dimension $7 \mathrm{~cm} \times 7 \mathrm{~cm}$ with a deviation angle of 30 arcsec fixed on one of the sub-apertures, the other is left open

- A digital CCD camera, operated in the visible (maximum sensitivity at $500 \mathrm{~nm}$ ) is at the telescope focus. The pixel size of $10 \mu \mathrm{m}$ is consistent with the spatial sampling of the Airy Disc of the sub-apertures. The camera is placed inside a thermostated box, ensuring a temperature never colder than $\geq-20^{\circ} \mathrm{C}$. Digital image signal was then transported by a $10 \mathrm{~m}$ cable to a PCI controler board inside a laboratory tent.

Nearly one month of seeing measurements were performed at Dome C, from Dec. 6, 2002 to January 2, 2003. The first days of the campaign were devoted to the precise polar alignment of the telescope mount. As the Sun was present 24 hours per day, we did the alignment by using the Bigourdan method on sunspots, Venus and Mercury. At local noon, with the Sun around $35^{\circ}$ above horizon, stars of magnitude 1 were still observable with a reasonable contrast. We selected Canopus $(\alpha$ Eri, $V=-0.9)$ for the seeing measurements.

\section{Data processing}

The main difficulty due to the daytime stellar observations is the sky background level. After different trials, an exposure time of $10 \mathrm{~ms}$ has finally been selected. It led to a background level of the order of 10 to $15 \%$ of the star image maximum intensity. 
A software was developed to perform real time data processing. Each sort exposure frame was thresholded to eliminate the background, then the two stellar images were easily detected and their photocenter coordinates computed by means of a simple barycenter formula.

Every two minutes, the variances of longitudinal and transverse distances between the two stars were computed on about 1000 individual images ("longitudinal" means parallel to the aperture alignment). These variances are obtained in unit of pixel square and require a calibration of the pixel size. That was done by imaging the double star Alpha Centauri, which angular separation $\rho=12 \operatorname{arcsec}$ has been estimated from the orbit of Heinz (1960)

Fried parameter was then computed from longitudinal $\left(\sigma_{1}^{2}\right)$ and transversal $\left(\sigma_{\mathrm{t}}^{2}\right)$ variances using Eqs. (5) and (8) of Tokovinin (2002). The wavelength is $\lambda=500 \mathrm{~nm}$. The two estimations of $r_{0}$ are expected to give similar values ; all measurements for which $\left|1-r_{0}^{(\mathrm{t})} / r_{0}^{(\mathrm{l})}\right|>0.1$ were rejected. The seeing is then calculated and compensated from the zenith angle effect by the standard formula $\epsilon^{(\mathrm{t} \mid \mathrm{l})}=0.98 \cos z^{-3 / 5} \lambda / r_{0}^{(\mathrm{tll})}$

The zenithal angle $z$ of Canopus at Dome $C$ ranges between $22^{\circ}$ and $52^{\circ}$, the minimum height being between 11 and $12 \mathrm{pm}$ local time (UT+8) during the observing month. At such a large value of $z$, the compensation formula in $\cos z^{-3 / 5}$ may be a little bit inaccurate.

Another point to be taken into account in the seeing estimation is the exposure time. The seeing must be ideally given for instantaneous images, and the finite exposure time is responsible for a blurring of the images that artificially improves the measured seeing. This effect has been extensively investigated (for instance see Tokovinin 2002; Ziad et al. 2000). The proposed solution for correcting it is to estimate its amplitude by artificially increasing the exposure time $\tau$ by binning the data. This was made on a few image sets sampled at 40 frames per second ; we computed the seeing for $\tau, 2 \tau, 3 \tau$ and $4 \tau$, and obtained the trend that can be extrapolated for $0 \tau$. This trend is close to an exponential behaviour and results, with our exposure time of $10 \mathrm{~ms}$, to a correction factor of the order of $5 \%$.

\section{Results}

During the first week (December 6th-13th), the telescope was at ground level, its mount just standing on the snow surface. Then a wooden platform was erected and starting on Dec. 16, the data have been collected from the top of this platform, 7 meters above the snow level, thus avoiding the always possible surface layer turbulence.

A total amount of 3650 2-min seeing values have been estimated. Figures 2 and 3 show the seeing time series day by day. Thanks to the presence of two observers, several long time series have been made possible, for instance the star has been observed almost without stop during 48 hours between the 16th and the 18th of December.

The histogram of seeing values (averaged between transversal and longitudinal) is plotted in Fig. 1. The median of all 3650 individual values is $1.20 \operatorname{arcsec}$ (with an exposure

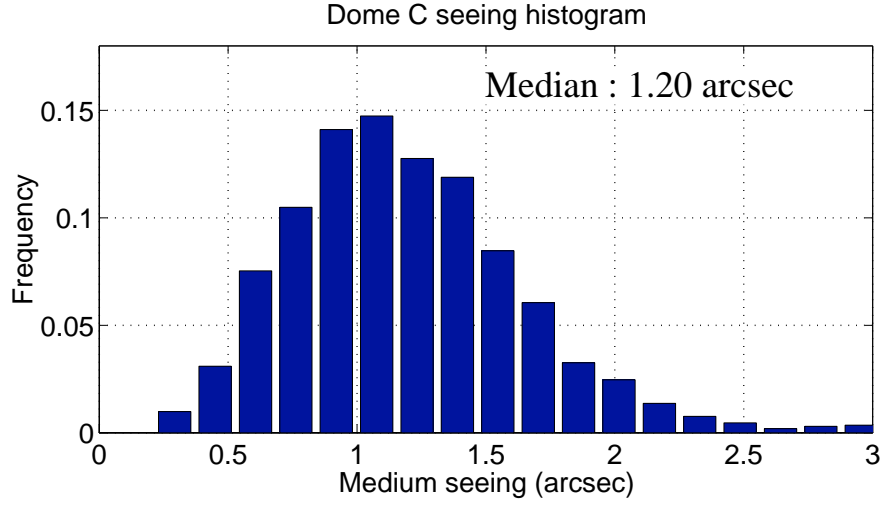

Fig. 1. Normalized histogram of seeing for an exposure time of $10 \mathrm{~ms}$. Transversal and longitudinal values have been averaged.

time of $10 \mathrm{~ms}$, the correction factor would then give an average of instant values of the order of $1.26 \mathrm{arcsec}$ ). $4 \%$ of individual measurements are better than 0.6 arcsec, $12 \%$ better than 0.76 arcsec and $22 \%$ better than 0.92 arcsec. Moreover several occurrences for long duration of weak turbulence are visible in Figs. 2 and 3. For instance, the seeing was consistently better than 1 arcsec during 5 hours on Dec. 17th, and again on Dec. 27 th.

\section{Discussion and conclusion}

These measurements are the very first DIMM data obtained on the Antarctic plateau. They are also the first DIMM measurements ever performed in daytime. Although our measurements were made with the Sun always present between $5^{\circ}$ and $35^{\circ}$ above horizon, the seeing values can almost compare to those of other well known astronomical sites (Ziad et al. 2000). The fact that the best seeing values, like 0.5 arcsec or better), have been generally obtained in mid local afternoon (look at Dec. 7, $10,17,19,20,21,28,31$ and Jan. 1) is extremely encouraging. Indeed, a discontinuity of the temperature gradient between 200 and $400 \mathrm{~m}$ has often been noted in the middle of the day, and disappears in the evening to be replaced by a standard surface inversion layer of 20 or $30 \mathrm{~m}$. During the afternoon transition, there is a moment with an isothermal temperature profile. The generally excellent seeing obtained during this transition indicates that the contribution of all the rest of the atmosphere is indeed very small. At night with a telescope standing above the ground inversion layer, a really excellent seeing can then be expected almost continuously. We are now working at the improved DIMM instrument suitable for night time operation at Dome $\mathrm{C}$ with ground temperatures of the order of $-70{ }^{\circ} \mathrm{C}$, during the first winterover scheduled for 2004 .

Acknowledgements. We wish to thank both Polar Insitutes, IPEV and ENEA, that made possible to have two observers on the site during this summer season, and all the Concordia staff at Dome $\mathrm{C}$ for their friendly and efficient help in setting up the Concordiastro platform. 


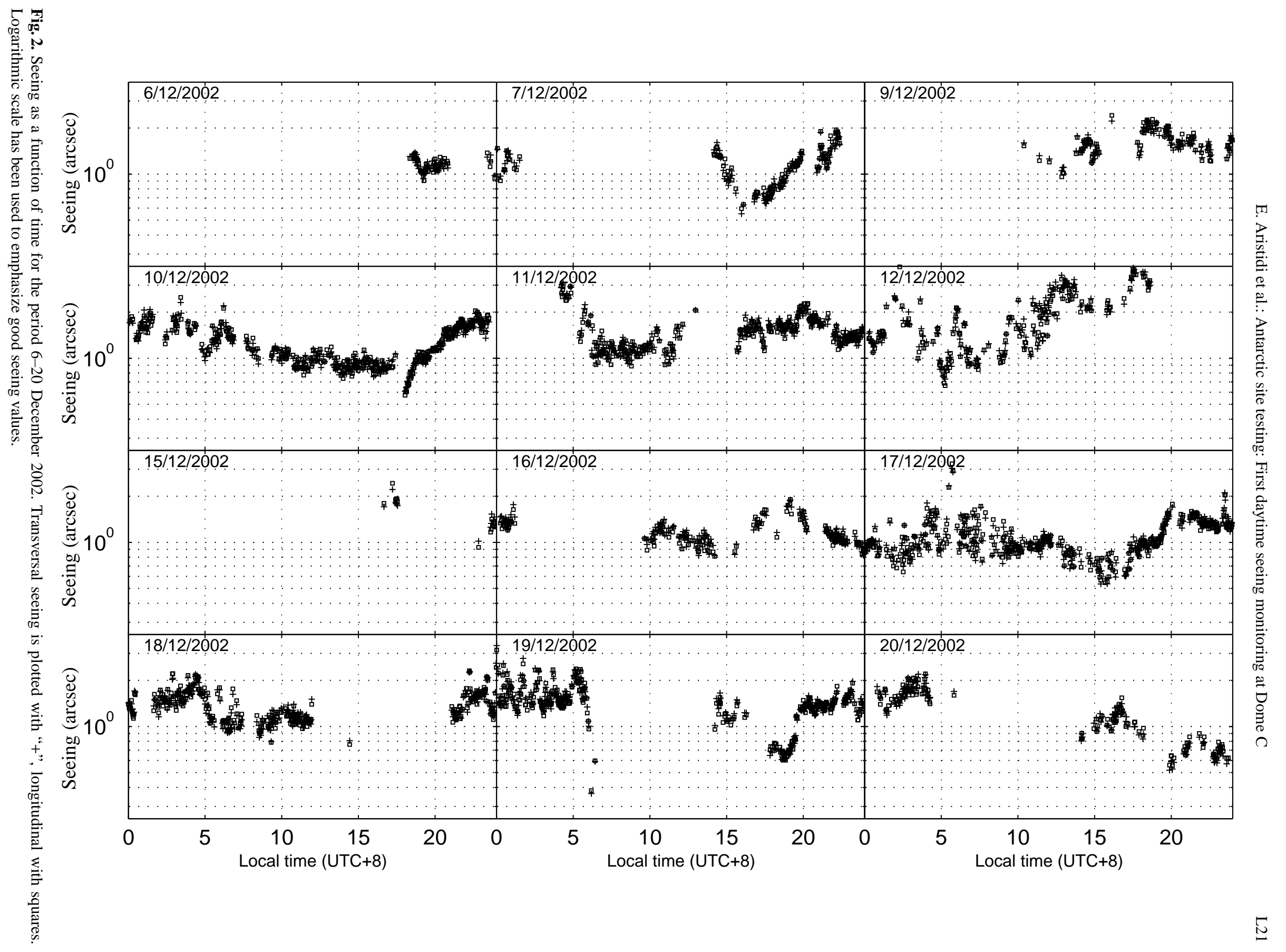




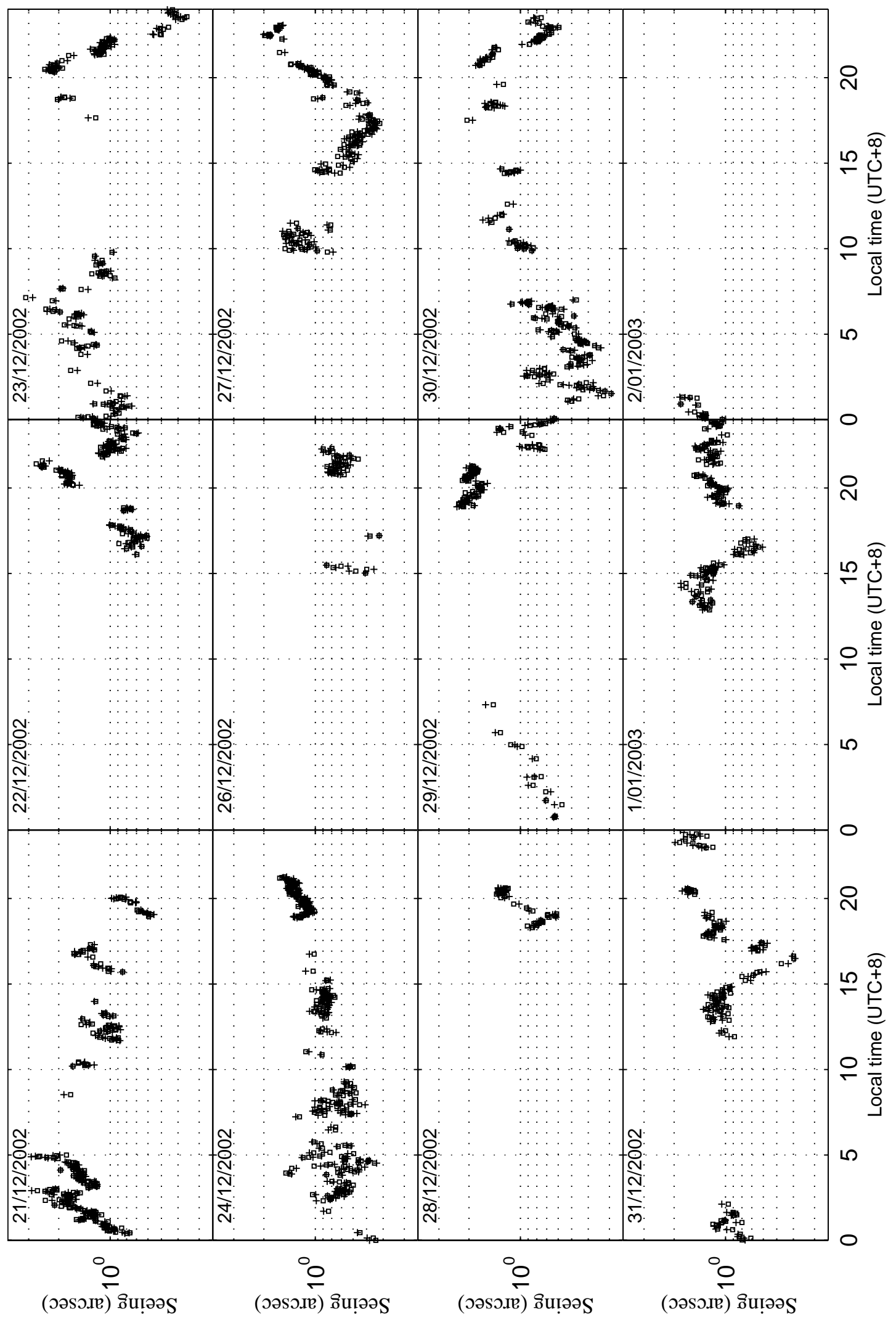

Fig. 3. Same Fig. 2 for the period 21 December 2002-1 January 2003.

\section{References}

Heintz, W. D. 1960, Veroff. Sternw. Munchen, 5, 100

Sarazin, M., \& Roddier, F. 1990, A\&A, 227, 294
Tokovinin, A. 2002, PASP, 114, 1156

Vernin, J., \& Muñoz-Tuñòn, C. 1995, PASP, 107, 265

Ziad, A., Conan, R., Tokovinin, A., Martin, F., \& Borgnino, J. 2000, Appl. Opt., 39, 5415 